\title{
Existence and Uniqueness of the Solution to the 3D Navier-Stokes Equations in the Homogeneous Sobolev-Gevrey Space.
}

\author{
Xiaochun Sun, Jia Liu
}

Abstract-We apply the principle of compression mapping to show existence and uniqueness of solutions for the classical Navier-Stokes equations in Sobolev-Gevrey space $\dot{H}_{a, \sigma}^{\frac{1}{2}}$.

Index Terms-Navier-Stokes equations; Sobolev-Gevrey spaces ;existence and uniqueness.

\section{INTRODUCTION}

The 3D generalized Navier-Stokes system is give by:

$\begin{cases}\partial_{t} u-\nu \Delta u=Q(u, u), & x \in \mathbb{R}^{3}, t \in(0, \infty), \\ u=0, & x \in \mathbb{R}^{3}, t \in(0, \infty), \\ u(0, x)=u^{0}(x), & x \in \mathbb{R}^{3},\end{cases}$

with $\mathrm{Q}$ is the bilinear operator defined as:

$\mathrm{Q}^{j}(u, v)=\sum_{k, l, m} q_{k, l}^{j, m} \partial_{m}\left(u^{k} v^{l}\right), \quad j=1,2,3$,

where

$\mathrm{q}_{k, l}^{j, m}=\sum_{n, p=1}^{3} a_{k, l}^{j, m, p, n} \mathcal{F}^{-1}\left(\frac{\xi_{n} \xi_{p}}{|\xi|^{2}} \hat{u}(\xi)\right)$

and $a_{k, l}^{j, m, p, n}$ are real numbers.

The particular case of the above system is the Navier-Stokes system for incompressible fluid:

$\begin{cases}\partial_{t} u-\nu \Delta u+u \cdot \nabla u=-\nabla p & x \in \mathbb{R}^{3}, t \in(0, \infty), \\ u=0, & x \in \mathbb{R}^{3}, t \in(0, \infty), \\ u(0, x)=u^{0}(x), & x \in \mathbb{R}^{3},\end{cases}$

where $v>0$ is the viscosity of the fluid, and $\alpha>0$ is real parameters. $u=(t, x)$ is the velocity field of fluid,

$p=p(t, x) \in \square$ denotes the unknow pressure of the fluid at the point $(t, x) \in \square^{+} \times \square^{3}$, and $(u \cdot \nabla u):=u_{1} \partial_{1} u+u_{2} \partial_{2} u+u_{3} \partial_{3} u$ , while $u^{0}=\left(u_{1}^{0}(x), u_{2}^{0}(\mathrm{x}), u_{3}^{0}(\mathrm{x})\right)$ is a given initial velocity. If $u^{0}$ is a quite regular, the divergence free condition determines the pressure $p$. Here $\partial_{t}$ and $\Delta=\sum_{j=1}^{n} \partial_{x_{j}}^{2}$ are the partial derivative with respect to $t$ and the Laplacian with respect to $x=\left(x_{1} \cdots, x_{n}\right)$. For simplicity, we will take $v=1$.

Xiaochun Sun, College of Mathematics and Statistics, Northwest Normal University, LanzhouGansu730070,China..

Jia Liu, College of Mathematics and Statistics, Northwest Normal University, Lanzhou Gansu 730070, China.
We are mainly interested in studying existence and uniqueness of solutions for the classical Navier-Stokes equations. Here we extend the results obtained in [1] to the case of homogeneous Sobolev-Gevrey space. To define the spaces, for $s \in \square$, let us denote by $H^{s}\left(\square^{3}\right)$ the usual Sobolev spacs on $\square^{3}$, with respective inner product $<,>_{H^{s}\left(\square^{3}\right)}$, and $\dot{H}^{s}\left(\square^{3}\right)$ denote the usual homogeneous Sobolev spacs on $\square^{3}$, with respective inner product $<,>_{\dot{H}^{s}\left(\square^{3}\right)}$. We denote the Fourier transform, as in [3] by $F(f)(\xi)=\int_{\square^{2}} \exp (-i x \cdot \xi) f(x) d x, \xi=\left(\xi_{1}, \xi_{2}, \xi_{3}\right) \in \square^{3}$, and its inverse by $F^{-1}(f)(x)=(2 \pi)^{-2} \int_{\square^{2}} \exp (i x \cdot \xi) f(\xi) d \xi, x=\left(x_{1}, x_{2}, x_{3}\right) \in \square^{3}$. The convolution product of a suitable pair of function $f$ and $\mathrm{g}$ on $\square^{3}$ is given by

$(f * g)(x)=\int_{\square} f(x-y) g(y) d y, x \in \square^{3}$

If $f=\left(f_{1}, f_{2}, f_{3}\right), g=\left(g_{1}, g_{2}, g_{3}\right)$ are two vector fields, we denote $f \otimes g:=\left(g_{1} f, g_{2} f, g_{3} f\right)$ and $\operatorname{div}(f \otimes g):=\left(\operatorname{div}\left(g_{1} f\right), \operatorname{div}\left(g_{2} f\right), \operatorname{div}\left(g_{3} f\right)\right)$. The fractional Laplace operator $(-\Delta)^{\frac{s}{2}}, 0<s<2$, is defined by

$\mathcal{F}\left((-\Delta)^{\frac{s}{2}}\right.$
We write $|D|=-\Delta^{\frac{1}{2}}$

Definition1.1

For $a, s>0, \sigma \geq 1$ and $|\mathrm{D}|=(-\Delta)^{\frac{1}{2}}$, The Sobolev-Gevery spaces $H_{a, \sigma}^{s}\left(\mathbb{R}^{3}\right)$ defined as follows:

$$
H_{a, \sigma}^{s}\left(\mathbb{R}^{3}\right)=\left\{f \in L^{2}\left(\mathbb{R}^{3}\right) ; e^{a|D|^{\frac{1}{\sigma}}} f \in H^{s}\left(\mathbb{R}^{3}\right)\right\}
$$

which is equipped with the norm

$$
\|f\|_{H_{a, \sigma}^{s}}=\left\|e^{a|D|^{\frac{1}{\sigma}}} f\right\|_{H^{s}}
$$

and the associated inner product

$$
\langle f / g\rangle_{H_{a, \sigma}^{s}}=\left\langle e^{a|D|^{\frac{1}{\sigma}}} f / e^{a|D|^{\frac{1}{\sigma}}} g\right\rangle_{H^{s}} .
$$

Analogously, the homogeneous Sobolev-Gevery spaces $\dot{H}_{a, \sigma}^{s}\left(\mathbb{R}^{3}\right)$ is

$$
\dot{H}_{a, \sigma}^{s}\left(\mathbb{R}^{3}\right)=\left\{f \in L^{2}\left(\mathbb{R}^{3}\right) ; e^{a|D|^{\frac{1}{\sigma}}} f \in \dot{H}^{s}\left(\mathbb{R}^{3}\right)\right\}
$$

which is equipped with the norm

$$
\|f\|_{\dot{H}_{a, \sigma}^{s}}=\left\|e^{a|D|^{\frac{1}{\sigma}}} f\right\|_{\dot{H}^{s}}
$$

and the associated inner product 


\section{Existence and Uniqueness of the Solution to the 3D Navier-Stokes Equations in the Homogeneous Sobolev-Gevrey Space}

$$
\langle f / g\rangle_{\dot{H}_{a, \sigma}^{s}}=\left\langle e^{a|D|^{\frac{1}{\sigma}}} f / e^{a|D|^{\frac{1}{\sigma}}} g\right\rangle_{\dot{H}^{s}} .
$$

Our main result is the following.

\section{Theorem.1.2}

Let $s>0, \sigma \geq 1$. If $u^{0} \in \dot{H}_{a, \sigma}^{\frac{1}{2}}$ such that $\operatorname{div} u^{0}=0$, then there exists a positive time $T$ such that (1.1) has unique solution $u \in L^{4}\left([\mathrm{O}, T], \dot{H}_{a, \sigma}^{1}\right)$ which also belongs to $u \in C\left([0, T], \dot{H}_{a, \sigma}^{\frac{1}{2}}\right) \cap L^{2}\left([0, T], \dot{H}_{a, \sigma}^{\frac{3}{2}}\right)$.

\section{PRELIMINARIES}

\section{Lemma.2.1 ${ }^{[5]}$}

Let $E$ be a Banach space, $B$ a continuous bilinear map from $E \times E \rightarrow E$, and a positive real number such that $\alpha<\frac{1}{4\|\mathrm{~B}\|}$, with

$$
\|B\|=\sup _{\|u\| \leq 1,\|v\| \leq 1}\|B(u, v)\| .
$$

For any $a$ in the ball $B(0, \alpha)$ in $E$, then there exists a unique $x$ in $B(0,2 \alpha)$ such that

\section{Lemma.2.2 ${ }^{[3]}$}

$$
x=a+B(x, x)
$$

Let $a>0, \sigma \geq 1$ and $\left(s_{1}, s_{2}\right) \in \mathbb{R}^{2}$, such that $s_{1}<\frac{3}{2}$, $s_{1}+s_{2}>0$. Then there exists a constant $C=C\left(s_{1}, s_{2}\right)$, such that for all $u, v \in \dot{H}_{a, \sigma}^{s_{1}}\left(\mathbb{R}^{3}\right) \cap \dot{H}_{a, \sigma}^{s_{2}}\left(\mathbb{R}^{3}\right)$, we have $\|u v\|_{\dot{H}_{a, \sigma}^{s_{1}+s_{2}-\frac{3}{2}}\left(\mathbb{R}^{3}\right)} \leq C\left(\|u\|_{\dot{H}_{a, \sigma}^{s_{1}}\left(\mathbb{R}^{3}\right)}\|v\|_{\dot{H}_{a, \sigma}^{s_{2}}\left(\mathbb{R}^{3}\right)}+\right.$ $\left.\|u\|_{\dot{H}_{a, \sigma}^{s_{2}}\left(\mathbb{R}^{3}\right)}\|v\|_{\dot{H}_{a, \sigma}^{s_{1}}\left(\mathbb{R}^{3}\right)}\right)$.

If $a>0, s_{1}<\frac{3}{2}, s_{2}<\frac{3}{2}$ and $s_{1}+s_{2}>0$, then exists a constant $C=C\left(s_{1}, s_{2}\right)$, such that $\|u v\|_{\dot{H}_{a, \sigma}^{s_{1}+s_{2}-\frac{3}{2}}\left(\mathbb{R}^{3}\right)} \leq C\|u\|_{\dot{H}_{a, \sigma}^{s_{1}}\left(\mathbb{R}^{3}\right)}\|v\|_{\dot{H}_{a, \sigma}^{s_{2}}\left(\mathbb{R}^{3}\right)}$.

\section{Lemma.2.3}

Let $a>0$ and $\sigma \geq 1$. Let $Q$ be a bilinear form as defined in (1.1). Then, there exists a constant $C>0$ such that for all $u, v \in \dot{H}_{a, \sigma}^{1}\left(\mathbb{R}^{3}\right)$ we have:

$$
\|Q(u, v)\|_{\dot{H}_{a, \sigma}^{-\frac{1}{2}}} \leq C\|u\|_{\dot{H}_{a, \sigma}^{1}}\|v\|_{\dot{H}_{a, \sigma}^{1}} .
$$

Proof Thanks to the inequality (2.1), we get:

$$
\begin{aligned}
\|Q(u, v)\|_{\dot{H}_{a, \sigma}^{-\frac{1}{2}}} & \leq C \sup _{k, l}\left(\left\|u^{k} \partial v^{l}\right\|_{\dot{H}_{a, \sigma}^{-\frac{1}{2}}}+\left\|v^{l} \partial u^{k}\right\|_{\dot{H}_{a, \sigma}^{-\frac{1}{2}}}\right) \\
& \leq C\left(\|u\|_{\dot{H}_{a, \sigma}^{1}}\|\nabla v\|_{\dot{H}_{a, \sigma}^{0}}+\|v\|_{\dot{H}_{a, \sigma}^{1}}\|\nabla u\|_{\dot{H}_{a, \sigma}^{0}}\right) \\
& \leq 2 C\|u\|_{\dot{H}_{a, \sigma}^{1}}^{1}\|v\|_{\dot{H}_{a, \sigma}^{1}} .
\end{aligned}
$$

\section{Lemma.2.4}

Let $u$ be the solution in $C\left([0, T], S^{\prime}\right)$ of the Cauchy problem

$$
\left\{\begin{array}{l}
\partial_{t} u-\Delta u=f \\
u(0, x)=u^{0}(x)
\end{array}\right.
$$

with and $u^{0} \in \dot{H}_{a, \sigma}^{\frac{1}{2}}$. Then

$$
u \in\left(\cap_{p=2}^{\infty} L^{p}\left([0, T], \dot{H}_{a, \sigma}^{\frac{1}{2}+\frac{2}{p}}\right) \cap C\left([0, T], \dot{H}_{a, \sigma}^{\frac{1}{2}}\right)\right) .
$$

Moreover, we have the following estimates:

$$
\begin{aligned}
& (2.1)\|u\|_{\dot{H}_{a, \sigma}^{\frac{1}{2}}}^{2}+\int_{0}^{t}\|\nabla u\|_{\dot{H}_{a, \sigma}^{\frac{1}{2}}}^{2} d s \leq\left\|u^{0}\right\|_{\dot{H}_{a, \sigma}^{\frac{1}{2}}}^{2}+\int_{0}^{t}\|f(s)\|_{\dot{H}_{a, \sigma}^{-\frac{1}{2}}}^{2} d s \\
& (2.2)\left[\int_{\mathbb{R}^{3}}|\xi| e^{2 a|\xi|^{\frac{1}{\sigma}}}\left(\sup _{0 \leq t^{\prime} \leq t}\left|\hat{u}\left(t^{\prime}, \xi\right)\right|\right)^{2} d \xi\right]^{\frac{1}{2}} \leq\left\|u^{0}\right\|_{\dot{H}_{a, \sigma}^{\frac{1}{2}}}+\|f\|_{L^{2}\left([0, t), \dot{H}_{a, \sigma}^{-\frac{1}{2}}\right)} \\
& (2.3)\|u\|_{L_{T}^{p}\left(\dot{H}_{a, \sigma}^{\frac{1}{2}+\frac{2}{p}}\right)} \leq\left\|u^{0}\right\|_{\dot{H}_{a, \sigma}^{\frac{1}{2}}}+\|f\|_{L^{2}\left([0, t), \dot{H}_{a, \sigma}^{-\frac{1}{2}}\right)}
\end{aligned}
$$

Proof The first estimate is just the energy estimate.

$$
\partial_{t} u-\Delta u=f \text {. }
$$

Let us take the inner product in $\dot{H}_{a, \sigma}^{\frac{1}{2}}$ with $u$, we get

$\frac{1}{2} \frac{d}{d t}\|u\|_{\dot{H}_{a, \sigma}^{\frac{1}{2}}}^{2}+\|\nabla u\|_{\dot{H}_{a, \sigma}^{\frac{1}{2}}}^{2} \leqslant|<f, u\rangle_{\dot{H}_{a, \sigma}^{\frac{1}{2}}}$

$$
\leqslant C\|u\|_{\dot{H}_{a, \sigma}^{\frac{1}{2}}}\|f\|_{\dot{H}_{a, \sigma}^{-\frac{1}{2}}}^{2}
$$

Taking the $L^{1}$ norm with respect to time and using Young's inequality, we deduce the results.

The proof of the second one is based around writing Duhamel's formula in Fourier space, namely,

$\hat{u}(t, \xi)=e^{-t|\xi|^{2}} \widehat{u^{0}}(\xi)+\int_{0}^{t} e^{-(t-s)|\xi|^{2}} \hat{f}(s, \xi) d s$

The Cauchy-Schwartz inequality implies that: For any $0<t^{\prime}<T$, we get

$\sup _{0 \leq t^{\prime} \leq t}\left|\hat{u}\left(t^{\prime}, \xi\right)\right| \leq \widehat{\mid u^{0}}(\xi) \mid+\frac{1}{\sqrt{2}|\xi|}\|\hat{f}(\xi, \cdot)\|_{L^{2}([0, t))}$.

Multiplying the obtained inequality by $|\xi|^{\frac{1}{2}} e^{a|\xi|^{\frac{1}{\sigma}}}$, we obtain

$|\xi|^{\frac{1}{2}} e^{a|\xi|^{\frac{1}{\sigma}}} \sup _{0 \leq t^{\prime} \leq t}\left|\hat{u}\left(t^{\prime}, \xi\right)\right| \leq|\xi|^{\frac{1}{2}} e^{a|\xi|^{\frac{1}{\sigma}}}\left|\widehat{u^{0}}(\xi)\right|+\frac{|\xi|^{\frac{1}{2}} e^{a|\xi|^{\frac{1}{\sigma}}}}{\sqrt{2}|\xi|}\|\hat{f}(\xi, \cdot)\|_{L^{2}([0, t))}$

Taking the $L^{2}$ norm with respect to the frequency variable $\xi$, we conclude that:

$\left[\int_{\mathbb{R}^{3}}|\xi| e^{2 a|\xi| \frac{1}{\sigma}}\left(\sup _{0 \leq t^{\prime} \leq t}\left|\hat{u}\left(t^{\prime}, \xi\right)\right|\right)^{2} d \xi\right]^{\frac{1}{2}} \leq\left\|u^{0}(\xi)\right\|_{\dot{H}_{a, \sigma}^{\frac{1}{2}}}+\|f\|_{L^{2}\left([0, t), \dot{H}_{a, \sigma}^{-\frac{1}{2}}\right)}$

Since, for almost all fixed $\xi \in \mathbb{R}^{3}$, the map $t \mapsto \hat{u}(t, \xi)$ is continuous over $[0, T]$, the Lebesgue dominated convergence theorem ensures that $u \in C\left([0, T], \dot{H}_{a, \sigma}^{\frac{1}{2}}\left(\mathbb{R}^{3}\right)\right)$.Similary, we have:

$|\xi|^{\frac{3}{2}} e^{a|\xi| \frac{1}{\sigma}}|\hat{u}| \leq|\xi|^{\frac{3}{2}} e^{-t|\xi|^{2}} e^{a|\xi| \frac{1}{\sigma}}\left|\widehat{u^{0}}\right|+\int_{0}^{t}|\xi|^{\frac{3}{2}} e^{-(t-s)|\xi|^{2}} e^{\left.a|\xi|\right|^{\frac{1}{\sigma}}}|\hat{f}(s, \xi)| d s$

Taking the $L^{2}$ norm with respect to time and using Young's inequality, we obtain:

$$
\begin{aligned}
{\left[\int_{0}^{t}|\xi|^{3} e^{2 a|\xi|^{\frac{1}{\sigma}}}|\hat{u}(\xi, s)|^{2} d s\right]^{\frac{1}{2}} } & \leqslant\left(\int_{0}^{t}|\xi|^{2} e^{-\left.2 s \xi \xi\right|^{2}} d s\right)^{\frac{1}{2}}|\xi|^{\frac{1}{2}} e^{a|\xi| \frac{1}{\sigma}} \widehat{\left|u^{0}\right|} \\
& +\int_{0}^{t}|\xi|^{2} e^{-s|\xi|^{2}} d s\left(\int_{0}^{t}|\xi|^{-1} e^{2 a|\xi| \frac{1}{\sigma}}|\hat{f}(s, \xi)|^{2} d s\right)^{\frac{1}{2}} \\
& \leqslant|\xi|^{\frac{1}{2}} e^{a|\xi| \frac{1}{\sigma}} \widehat{\mid u^{0}} \mid+\left(\int_{0}^{t}|\xi|^{-1} e^{2 a|\xi \xi| \frac{1}{\sigma}}|\hat{f}(s, \xi)|^{2} d s\right)^{\frac{1}{2}}
\end{aligned}
$$

Taking the $L^{2}$ norm with respect to the frequency variable $\xi$, we obtain :

$$
\|u\|_{L_{T}^{2}\left(\dot{H}_{a, \sigma}^{\frac{3}{2}}\right)} \leq\left\|u^{0}\right\|_{\dot{H}_{a, \sigma}^{\frac{1}{2}}}+\|f\|_{L_{T}^{2}\left(\dot{H}_{a, \sigma}^{-\frac{1}{2}}\right)}
$$

- Finally, the last inequality follows by interpolation:

and

$$
\|u\|_{\dot{H}_{a, \sigma}^{\frac{1}{2}+\frac{2}{p}}} \leq\|u\|_{\dot{H}_{a, \sigma}^{\frac{1}{2}}}^{1-\frac{2}{2}}\|u\|_{\dot{H}_{a, \sigma}^{\frac{3}{2}}}^{\frac{2}{2}}
$$

$$
\|u\|_{\dot{H}_{a, \sigma}^{\frac{1}{2}+\frac{2}{p}}}^{p} \leq\|u\|_{\dot{H}_{a, \sigma}^{\frac{1}{2}}}^{p-2}\|u\|_{\dot{H}_{a, \sigma}^{\frac{3}{2}}}^{2}
$$

Taking the $L^{1}$ norm with respect to time and using the inequalities (2.2) and (2.4) lead to (2.3).

\section{PROOF OF THEOREM 1.1}

Proof Let $B(u, u)$ be the solution to the heat equations.

$$
\left\{\begin{array}{l}
\partial_{t} B(u, u)-\Delta B(u, u)=Q(u, u) \\
B(u, u)=0 \\
B(u, u)(0)=0
\end{array}\right.
$$

with the bilinear operators $Q$ defined as in (1.2) and $\left.B(u, u)=-\int_{0}^{t} e^{-(t-s)(\Delta)} Q(u, u)\right) d s$ 
Thanks to Lemma 2.3, we have

$\int_{0}^{T}\|Q(u, v)\|_{\dot{H}_{a, \sigma}^{-\frac{1}{2}}}^{2} d s \leq C\|u\|_{L_{T}^{4}\left(\dot{H}_{a, \sigma}^{1}\right)}\|v\|_{L_{T}^{4}\left(\dot{H}_{a, \sigma}^{1}\right)}$

Thus, combining Duhamel's formula and the inequality (2.3), we have

$\|B(u, u)\|_{L_{T}^{4}\left(\dot{H}_{a, \sigma}^{1}\right)} \leq C\|u\|_{L_{T}^{4}\left(\dot{H}_{a, \sigma}^{1}\right)}\|u\|_{L_{T}^{4}\left(\dot{H}_{a, \sigma}^{1}\right)}$

this implies:

$$
\|B\|_{L_{T}^{4}\left(\dot{H}_{a, \sigma}^{1}\right)} \leq C<C_{0}
$$

thanks to Minkowski's inequality, we have

$$
\left\|e^{t \Delta} u^{0}\right\|_{L_{T}^{4}\left(\dot{H}_{a, \sigma}^{1}\right)} \leq\left\|u^{0}\right\|_{\dot{H}_{a, \sigma}^{\frac{1}{2}}}
$$

thus, if $\left\|u^{0}\right\|_{\dot{H}_{a, \sigma}^{\frac{1}{2}}} \leq \frac{1}{4 C_{0}}$, we get

$$
\left\|e^{t \Delta} u^{0}\right\|_{L_{T}^{4}\left(\dot{H}_{a, \sigma}^{1}\right)} \leq \frac{1}{4 C_{0}}<\frac{1}{4\|B\|} .
$$

According to Lemma 2.1, there exists a unique solution of (1.2) in the ball with center 0 and radius $\frac{1}{2 C_{0}}$ in the space $L^{4}\left([0, T] ; \dot{H}_{a, \sigma}^{1}\right)$ such that

$$
u(t, x)=e^{t \Delta} u^{0}+B(u, u) .
$$

we now consider the case of a large initial data $u^{0} \in \dot{H}_{a, \sigma}^{\frac{1}{2}}$. Let $\rho_{u_{0}}>0$, such that

$$
\left(\int_{|\xi| \geq \rho_{u_{0}}} e^{2 a|\xi|^{\frac{1}{\sigma}}}|\xi|\left|\widehat{u^{0}}\right|^{2} d \xi\right)^{\frac{1}{2}}<\frac{1}{8 C_{0}} .
$$

Using the inequality (3.2) and defining $v_{0}=\mathcal{F}^{-1}\left(\chi_{|\xi|<\rho_{u_{0}}} \widehat{u^{0}}\right)$, we get

$$
\begin{aligned}
\left\|e^{t \Delta} u^{0}\right\|_{L_{T}^{4}\left(\dot{H}_{a, \sigma}^{1}\right)} & \leqslant \| e^{t \Delta} \mathcal{F}^{-1}\left(\chi_{|\xi| \geq \rho_{u_{0}}} \widehat{\left.u^{0}\right)}\left\|_{L_{T}^{4}\left(\dot{H}_{a, \sigma}^{1}\right)}+\right\| e^{t \Delta} v_{0} \|_{L_{T}^{4}\left(\dot{H}_{a, \sigma}^{1}\right)}\right. \\
& \leqslant \frac{1}{8 C_{0}}+\left\|e^{t \Delta} v_{0}\right\|_{L_{T}^{4}\left(\dot{H}_{a, \sigma}^{1}\right)}
\end{aligned}
$$

From which we can deduce that

$$
\begin{aligned}
\left\|e^{t \Delta} v_{0}\right\|_{L_{T}^{4}\left(\dot{H}_{a, \sigma}^{1}\right)}^{4} & \leqslant \int_{0}^{T}\left[\int_{|\xi|<\rho_{u_{0}}}|\xi|^{2} e^{2 a|\xi|^{\frac{1}{\sigma}}}\left|\widehat{u^{0}}\right|^{2} d \xi\right]^{2} d t \\
& \leqslant \rho_{u_{0}}^{2} \int_{0}^{T}\left[\left.\int_{|\xi|<\rho_{u_{0}}}|\xi|\right|^{\frac{1}{\sigma}} e^{2 a|\xi|}\left|\widehat{u^{0}}\right|^{2} d \xi\right]^{2} d t \\
& \leqslant T \rho_{u_{0}}^{2}\left\|u^{0}\right\|_{\dot{H}_{a, \sigma}^{\frac{1}{2}}}^{4}
\end{aligned}
$$

which yields

$$
\left\|e^{t \Delta} v_{0}\right\|_{L_{T}^{4}\left(\dot{H}_{a, \sigma}^{1}\right)} \leq\left(T \rho_{u_{0}}^{2}\right)^{\frac{1}{4}}\left\|u^{0}\right\|_{\dot{H}_{a, \sigma}^{\frac{1}{2}}}
$$

Thus, if

$$
T \leq\left(\frac{1}{8 C_{0} \rho_{u_{0}}^{\frac{1}{2}}\left\|u^{0}\right\|_{\dot{H}_{a, \sigma}^{\frac{1}{2}}}}\right)^{4},
$$

then we conclude the existence of a unique solution in the ball with center 0 and radius $\frac{1}{2 C_{0}}$ in the space $L^{4}\left([0, T] ; \dot{H}_{a, \sigma}^{1}\right)$ and we observe that if $u$ is a solution of (1.2) in $L^{4}\left([0, T] ; \dot{H}_{a, \sigma}^{1}\right)$, then, by Lemma $2.3 Q(u, u)$ belongs to $L_{T}^{2}\left(\dot{H}_{a, \sigma}^{-\frac{1}{2}}\right)$. Hence, Lemma 2.4 implies that the solution $u$ belongs to $C\left([0, T] ; \dot{H}_{a, \sigma}^{1}\right) \cap L^{2}\left([0, T] ; \dot{H}_{a, \sigma}^{\frac{3}{2}}\right)$.

\section{REFERENCES}

[1] Hajer Bahouri, J.Y Chemin and R. Danchin, Fourier Analysis and Nonlinear Partial Differential Equations, Springer Verlag, 343p, 2011.203-212.

[2] H. Bae, Existence and Analyticity of Lei-Lin Solution to the Navier-Stokes Equations, Proc. Amer. Math. Soc. 143 (2015), no. 7, 28872892

[3] J. Benameur, L.Jlali, On the blow up criterien of 3D-NSE in Sobolev Gevery spaces, Journal of Mathematical Fluid Mechanics 18(4)(2015).
[4] J. Benameur, L.Jlali, Long time decay for 3D Navier-Stokes equations in Sobolev-Gevery spaces, Electronic Journal of Differential Equation, (2016)N 104, pp 1-13.

[5] Z.Zhang, Z.Yin. Global well-posedness for the generalized Navier Stokes system. arXiv:1306.3735v1 [Math.Ap]17 june 2013.

[6] J. Y. Chemin; About Navier-Stokes Equations, Publication du laboratoire Jaques-Louis Lions, Universit de Paris VI, 1996, R96023.

[7] J. Y. Chemin, Le systme de NavierStokes incompressible soixante dix ans aprs Jean Leray, Actes des Journes Mathmatiques la Mmoire de Jean Leray, Smin. Congr. vol. 9, Soc. Math. France, Paris 2004, 99-123 — MR 2145938 - Zbl 1075.35035

[8] H.Fujita,T.Kato, On the Navier-Stokes initial value problem I.Arch.Ration.Mech.Anal.16 (1964) 269- 315

[9] I. Gallagher, D. Iftimie, F. Planchon, Non-blow up at large times and stability for global solutions to the NavierStokes equations, C. R. Acad. Sci. Paris, Ser. I 334 (2002) 289292.

[10] E.Hopf. Uber die Anfangswertaufgabe fur die hydrodinamischen Grundgleichungen, Math. Nachr.4 (1951) 213-231.

[11] J.Lery. Essai sur lr movement d'un liquide visqueus emplissant l'espace, Acta Math. 63(1933)22-25.

[12] J. Leray Sur le mouvement d'un liquide visqueux emplissant l'espace Acta Math., 63 (1) (1934), pp. 193-248.

[13] Z. Lei , F. Lin, Global Mild Solutions of Navier-Stokes Equations. Communications on Pure and Applied Mathematics, (2011). 1297-1304.

[14] Q. Liu, Y. Jia, B. Dong, Remarks on the weakstrong uniqueness for the 2D quasi-geostrophic equation in BMO space Applied Mathematics Letters, 25 (2012) 1470-1474.

[15] T.Kato, Quaasi-Linear Equations of Evolution, with Application to Partial Differential Equations, Lecture Notes in Math, vol. 448, Spring-Verlag, 1975, pp.25-70.

[16] J. Benameur, L.Jlali, Long time decay of 3D-NSE in Lei-Lin Gevery spaces. arXiv 1502.04197v1 [math.AP] 14 Feb 2015.

[17] HAJER ORF, Long time decay for global solution to the Navier-Stokes equations in Sobolev-Gevery spaces.arXiv:1903.03034v1 [math.AP] 7 Mar 2019. 